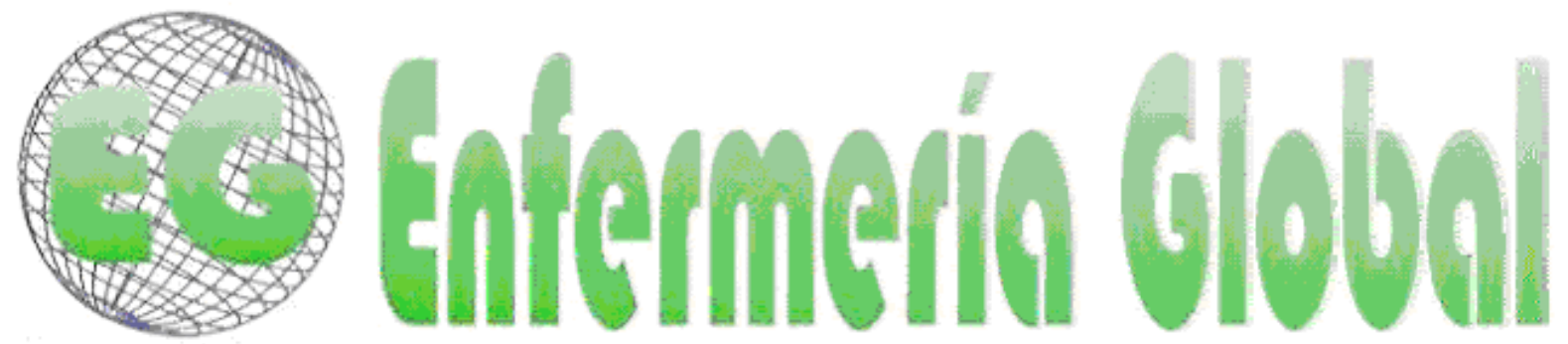

\title{
CLINICA
}

\section{Las relaciones personales que se establecen por los residentes de un hogar para ancianos.}

The personal relationships established by residents of a nursing home

\author{
*Blanca Gutiérrez, JJ. ${ }^{* *}$ Linares Abad, M. ${ }^{* * *}$ Grande Gascón, ML. \\ ${ }^{* * * * A r a n d a ~ M a r i ́ n, ~ D J . ~}$
}

\begin{abstract}
*Enfermero, Máster y Doctor en Gerontología Social por la Universidad de Jaén. Responsable de investigación del Hospital "Infanta Margarita" (Córdoba, España). E.mail: iiblanca@ephaq.es **Enfermero. Dr. en Antropología Social y Cultural. Profesor titular de la Facultad de Ciencias de la Salud. Universidad de Jaén. ${ }^{* * * E n f e r m e r a, ~ S o c i o ́ l o g a ~ y ~ D r a . ~ e n ~ C i e n c i a ~ P o l i ́ t i c a . ~ P r o f e s o r a ~ t i t u l a r ~ d e ~ l a ~}$ Facultad de Ciencias de la Salud. Universidad de Jaén. ****Enfermero de la Agencia Sanitaria Alto Guadalquivir. Sistema Sanitario Público de Andalucía.
\end{abstract}

Palabras clave: anciano; anciano de 80 o más años; hogares para ancianos; Investigación Cualitativa. Keywords: elderly; aged 80 and over; nursing homes; Qualitative Research.

\section{RESUMEN}

Objetivo: El propósito de este trabajo es estudiar y teorizar sobre las relaciones personales que se establecen en el seno de los hogares para ancianos.

Metodología: Estudio cualitativo de tipo interpretativo construido a partir del desarrollo de teoría fundamentada. El lugar de realización han sido los 6 hogares para ancianos de la comarca de la campiña sur de Córdoba, en el sur de España. Los participantes han sido 16 residentes y 4 profesionales de estos centros. La recogida de información se ha realizado a través de entrevistas en profundidad.

Resultados: En el contexto de las residencias de personas mayores hay principalmente tres grupos humanos que interaccionan con el mayor: los compañeros residentes, el compañero o compañeros de cuarto (si comparten dormitorio) y las personas que lo cuidan. Se han construido una serie de roles en cada una de estos grupos de convivientes, para la cual nos vamos a basar en la dimensionalización de algunas características de estas personas. La situación en uno u otro de estos roles no es estática ni permanente, las personas pueden ir cambiando de unos a otros en función de las diversas circunstancias. 


\title{
ABSTRACT
}

Objective: The purpose of this paper is to study and develop a theory about the personal relationships established within nursing homes.

\begin{abstract}
Methodology: qualitative and interactive investigation built from the development of a grounded theory. The study took place in 6 nursing homes in the region of the southern districts of Cordoba, in southern Spain. The participants were 16 residents and 4 professionals of these centers. The collection of information was done through in depth interviews.
\end{abstract}

Results: In the context of the nursing homes there are mainly three groups of people who interact with the elderly: partners, roommates (when sharing a bedroom) and caregivers. We have built some roles in each of these groups living together, on which we will base the dimensioning of some of the characteristics of these people. The situation in either of these roles is not static or permanent, people may change from one to another depending on various circumstances.

\section{ANTECEDENTES. ESTUDIOS CUALITATIVOS SOBRE LAS RELACIONES PERSONALES QUE SE ESTABLECEN EN UN HOGAR PARA ANCIANOS.}

\section{Relaciones de amistad entre los compañeros}

Bergland y Kirkevold ${ }^{(1)}$ realizaron un estudio en el que analizaron cómo influyen las relaciones estrechas entre compañeros en el concepto de "thriving" (bienestar psíquico y satisfacción emocional). Para que estas relaciones contribuyan a este tipo concreto de bienestar deben ser percibidas por los protagonistas como positivas y significativas. Aunque la relación compañero-compañero no era indispensable para que los residentes y las residentes experimentaran su satisfacción emocional, algunos de ellos manifestaron deseos de establecer una relación de este tipo. Concluían los autores que el hecho de que las personas cuidadoras desarrollen su rol como facilitadores de interacciones humanas significativas, es esencial para que se fortalezca esa experiencia de desarrollo.

Silva y cols. ${ }^{(2)}$ abordaron el tema de la amistad en una residencia de Brasil. Realizaron entrevistas a 15 personas mayores y utilizaron también la observación participante. Los participantes definían el concepto de amigo en función del apoyo que le proporcionaban las relaciones de amistad con respecto a las carencias que experimentaban en su vida diaria. En este contexto, las relaciones de amistad fueron más allá de las diferencias físicas, de edad o de cultura.

\section{La convivencia en los salones comunes y los dormitorios}

Hauge y Heggen ${ }^{(3)}$ usaron de la observación participante y entrevistaron a 5 residentes de dos instituciones de Noruega para investigar de qué manera los residentes consideraban como un hogar a su institución. Muchos mayores pasaban gran parte de su tiempo en los salones comunes. Los límites bien definidos es lo que para ellos definía a un hogar y estos salones comunes aunque también tenían ciertos límites físicos, éstos son considerados como ambiguos, por lo que los salones estarían situados en una especie de esfera intermedia entre lo público y lo privado. Las relaciones que se establecían en estos salones comunes fueron definidas como débiles y forzadas y se observaba cómo los residentes con cierta independencia preferían no estar en estas salas. Aunque los mayores incluidos en este estudio tenían habitaciones individuales decoradas y conformadas según sus gustos, consideraban difícil llevar un estilo de vida con privacidad en estas residencias. 
Oh ${ }^{(4)}$, estudió en una residencia de Corea mediante entrevistas en profundidad, las experiencias de 14 personas mayores mentalmente lúcidas que habían sufrido una apoplejía y que compartían su dormitorio con pacientes afectados de demencia. Los informantes relataban estar afectados por intensas y severas molestias. Con el tiempo se producía una evolución a través de la cual las personas mayores lúcidas intentaban encontrar un sentido a la situación a la que se estaban enfrentando y se pasaba a una sensación de resignación al comprender que no gozaban de potestad para cambiar la política de la institución. Se producían cambios en su carácter, sentían un miedo continuo a sufrir también de demencia, llegaban a infravalorar sus propias vidas, se volvían más indiferentes hacia los otros y perdían motivación para realizar sus actividades, lo cual se traducía finalmente en una disminución de su independencia.

\section{Relaciones de pareja}

Mendoza y cols. ${ }^{(5)}$, a través de 42 entrevistas semiestructuradas abordaron el tema de la sexualidad con las personas mayores de varias residencias de la provincia de Álava, en España. La vivencia sexual pasaba a lo largo de la vida de los informantes por dos etapas, ambas influenciadas por las creencias religiosas: en la primera, la sexualidad estaba claramente dirigida hacia la procreación, en la segunda etapa, la sexualidad se relacionaba con el cariño, con el afecto, con la tranquilidad y con el hecho de no estar solos. También informaban de que el hecho de no disponer de habitaciones individuales condicionaba en gran parte la vivencia de la intimidad.

Nay ${ }^{(6)}$, abordó también el tema de la sexualidad para lo cual entrevistó a 20 mujeres mayores residentes en una institución de Australia. Algunas comentaban que ya eran demasiado mayores para estos asuntos y que la sexualidad no era apropiada para las mujeres que no tenían marido. Definían la sexualidad como algo limitado a ser complacientes con su hombre y la asociaban con la reproducción y la belleza juvenil. Otras en cambio reconocían que sí pensaban en el sexo pero que no tenían oportunidades, lo cual lo atribuían a que había pocos hombres disponibles. Lo que echaban de menos era la cercanía, el toque de alguien especial y los abrazos estrechos en la cama durante la noche.

\section{La relación con los cuidadores}

Los participantes del estudio de Anderberg y Berglund (7), consideraban que los cuidados de los profesionales son determinantes para: mantener un equilibrio entre el dolor y el bienestar, conservar el control de su propio cuerpo, vivir el presente sin preocuparse en demasía por el futuro y ocultar la vulnerabilidad propia ante una situación que consideraban comprometida.

Chang ${ }^{(8)}$, estudió las percepciones referentes al autocuidado de personas mayores que vivían en centros de este tipo. Las creencias de los entrevistados, las expuso organizadas en torno a tres grandes temas: la importancia de realizar autocuidados, las barreras que impiden recuperar la capacidad de autocuidarse y los aspectos facilitadores que permiten recuperar esta capacidad.

Liukkonen (9), expuso a través de los participantes de su estudio cómo los profesionales estaban siempre muy ocupados y presionados por el tiempo, por lo que las personas mayores institucionalizadas creían necesario que los cuidadores 
pudieran hablar un poco más con ellos sobre sus esperanzas, sus deseos y sobre sus pensamientos diarios.

\section{OBJETIVOS DE LA INVESTIGACIÓN}

Con este trabajo se pretende ofrecer una visión teórica-interpretativa sobre las relaciones personales que establecen los residentes que viven en un hogar para ancianos.

\section{METODOLOGÍA}

La investigación consiste en un estudio cualitativo de tipo interpretativo, basado en el desarrollo de teoría fundamentada. ${ }^{(10)}$

El lugar de recogida de la información han sido los 6 hogares para ancianos de la comarca de la campiña sur de Córdoba, en el sur de España. La selección de los informantes ha consistido en un muestreo de tipo intencional y teórico ${ }^{(10,11)}$ : se ha invitado a participar en las entrevistas a las personas consideradas como más apropiadas para comprender el fenómeno de estudio, en este caso concreto, a personas mayores residentes y profesionales de hogares para ancianos. Paralelamente a la ejecución del análisis de datos, se han incluido más participantes con el objetivo de completar los códigos y categorías emergentes.

Cuando a pesar de realizar más entrevistas, el análisis de datos concurrente no proporcionaba propiedades y dimensiones inéditas para cada una de las categorías en estudio, no se incorporaron más informantes: criterio de saturación teórica ${ }^{(10,11)}$.

Las propias directoras de los centros, así como las enfermeras y profesionales de los hogares para ancianos asumieron la figura de "porteros", propia de la investigación cualitativa, permitiéndonos el desarrollo de la técnica de "bola de nieve" (12)

Se han realizado entrevistas en profundidad a un total de 16 residentes de estos centros: 7 hombres y 9 mujeres. La edad de las personas mayores entrevistadas ha oscilado desde los 56 hasta los 98 años y el tiempo que llevan viviendo en el hogar para ancianos ha variado desde 8 días hasta 26 años. No hemos incluido como participantes a personas mayores que estuvieran afectadas por enfermedades neurológicas degenerativas en estado avanzado. Así mismo, se ha entrevistado también a 4 profesionales cuidadores de estos centros.

Los encuentros se mantenían en una sala preparada para que los residentes puedan recibir a sus visitas o bien en los propios dormitorios de los informantes. Los testimonios de los participantes fueron grabados en formato digital a través de un dispositivo tipo MP3 y posteriormente transcritos literalmente.

Para desarrollar la teoría fundamentada se necesita del apoyo de diversas estrategias. Del muestreo teórico y de la saturación teórica ya se ha hablado anteriormente. Otro aspecto básico es el método de la comparación constante: se trata de simultáneamente codificar y analizar los datos con el propósito de desarrollar conceptos. Consiste en comparar continuamente los incidentes (unidades mínimas de análisis) de los datos para refinar conceptos, identificar sus propiedades, explorar cómo se relacionan entre sí e integrarlos en una teoría $(10,13,14)$ 
Otros recursos empleados han sido los memos o memorandos (anotaciones de ideas teóricas tal y como al investigador le surgía en el proceso de codificación) y los diagramas (representaciones gráficas de las relaciones entre conceptos).

Las tres etapas que han basado el análisis de datos han sido la codificación abierta, la codificación axial y la codificación selectiva ${ }^{(10,13,14)}$

En base a los criterios desarrollados por Emmanuell, Wendler y Grady (15), posteriormente adaptados al campo de la investigación cualitativa por Barrio y Simón

${ }^{(16)}$, hemos tratado de garantizar la corrección ética de nuestra investigación:

- Valor social o científico.

- Diseño metodológico debe ser coherente con los objetivos, seleccionando correctamente los contextos y los informantes. También la interpretación de los resultados.

- Cociente entre riesgo y beneficio favorable.

- Consentimiento informado firmado por cada uno de los participantes.

- Respeto por los participantes: protección de la intimidad y la confidencialidad. Se informó a los entrevistados de la forma en que se protegerán los datos, cómo se almacenarán y quién accederá a ellos.

- Evaluación independiente, que no ha de seguir los esquemas de evaluación típicos de los ensayos clínicos con medicamentos.

- Selección equitativa de los participantes.

Para trabajar y pretender asegurar el rigor científico de este trabajo, se han considerado una serie de criterios clave que según los diversos autores fundamentan la calidad científica de la investigación de tipo cualitativo: credibilidad, transferibilidad, confiabilidad y confirmabilidad ${ }^{(17,18)}$. Los criterios específicos tratados para apoyar la calidad científica de esta teoría fundamentada son cuatro: el ajuste de los datos, funcionamiento de la teoría, relevancia y modificabilidad ${ }^{(19,20)}$

\section{RESULTADOS}

En el contexto de las residencias de personas mayores hay principalmente tres grupos humanos que interaccionan con el mayor: los compañeros residentes, el compañero o compañeros de cuarto (si comparten dormitorio) y las personas que lo cuidan. Vamos a tratar de designar una serie de roles en cada una de estos convivientes, para lo cual nos vamos a basar en la dimensionalización de algunas características de estas personas. La situación en uno u otro de estos roles no es estática ni permanente, las personas pueden ir cambiando de unos a otros en función de las diversas circunstancias.

\section{Los compañeros residentes}

Vamos a dimensionar los conceptos de la carga afectiva y la pertenencia a un mismo estatus social-educativo (Figura 1). 
Figura 1. Los compañeros residentes

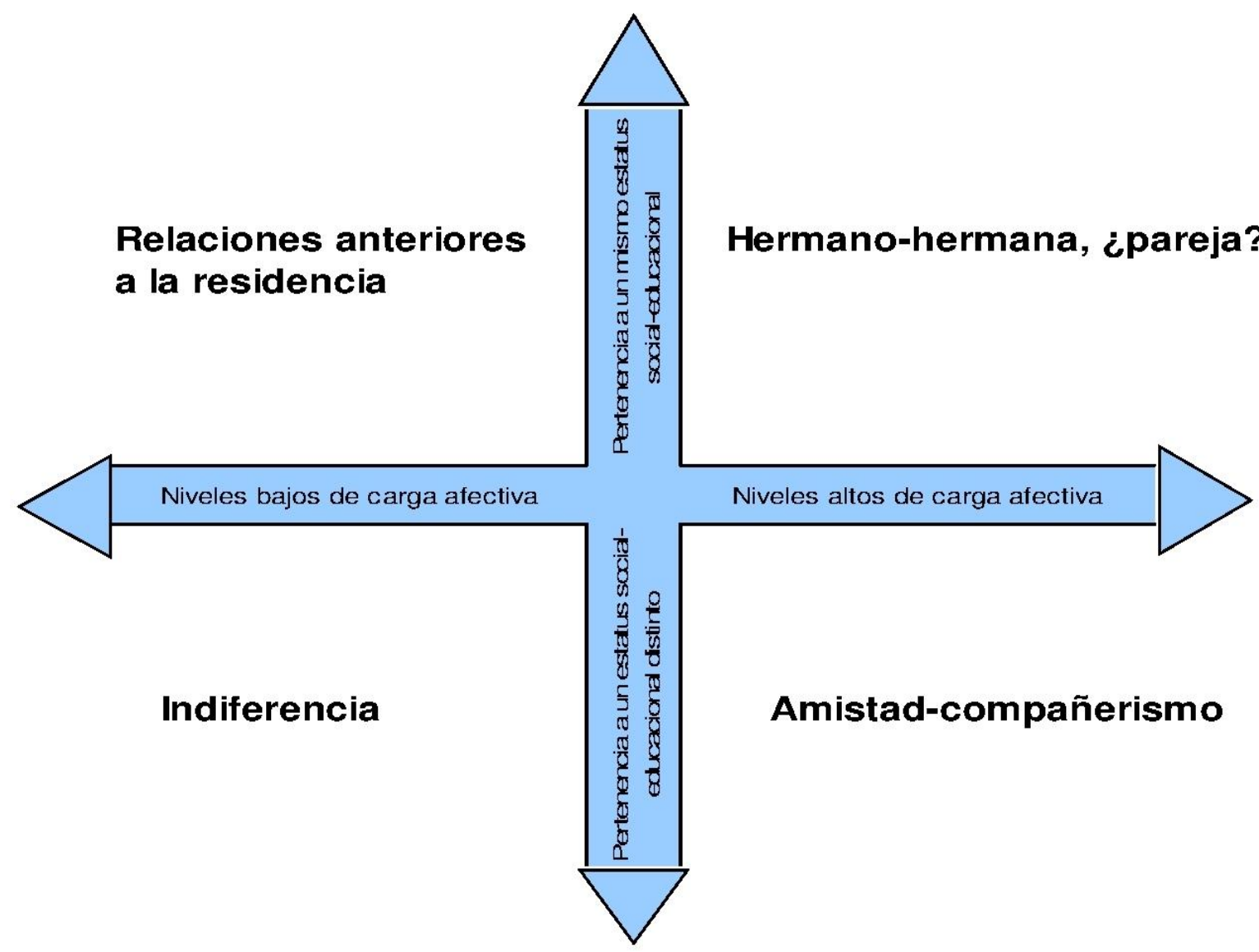

Niveles altos de carga afectiva-pertenencia a un mismo estatus social y educativo: se trata de compañeros a los que la persona mayor cataloga como "hermanos" o "hermanas" (códigos "in vivo"). Con ellos comparten todos los aspectos que pueden de la vida diaria como el ocio, las salidas a la calle o espacios físicos como la mesa en el comedor. En ocasiones se trata de relaciones que se habían formado ya desde la juventud. Cuando los niveles altos de estas dos características se dan en personas de distinto sexo, se puede llegar incluso a la constitución de parejas emocionales.

H6: "Me gusta escucharle y con él comemos a mediodía juntos y hay otro más, ahi en la mesa en el comedor. Con ése charlo más que con todos."

Niveles altos de carga afectiva-pertenencia a un estatus social y educativo distinto: se trata de "amigos" o "compañeros" (códigos "in vivo"), con los que se tienen encuentros ocasionales, manteniendo una actitud y un trato cordial y amable, pero con los que no se pretende compartir todas las circunstancias posibles de la vida diaria.

Niveles bajos de carga afectiva-pertenencia a un mismo estatus social y educativo: en muchas ocasiones se trata de residentes que se conocían desde la juventud y entre los que se ha mantenido algún tipo de rencilla o desencuentro a lo largo de los años, o incluso en la propia residencia de personas mayores. 
Niveles bajos de carga afectiva-pertenencia a un estatus social y educativo distinto: ante estos compañeros hay una actitud de indiferencia, si bien con el paso del tiempo se puede evolucionar al rol de amigo-compañero si se produce un aumento de la carga afectiva. A veces, el escaso apego afectivo se debe a conflictos surgidos por no respetar los valores de los otros grupos pertenecientes a un estatus distinto.

\section{El compañero o compañera de cuarto.}

Vamos a dimensionar los conceptos de carga afectiva y la ejecución de cuidados por parte de los convivientes en el cuarto (Figura 2).

Figura 2. El compañero o compañera de dormitorio.

Compañero-objeto Cuidado unidireccional

Compañero como molestia

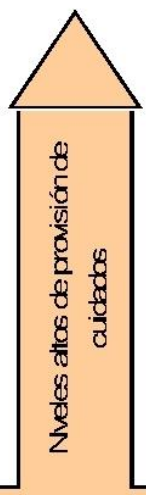

Hermano-hermana Cuidado mutuo

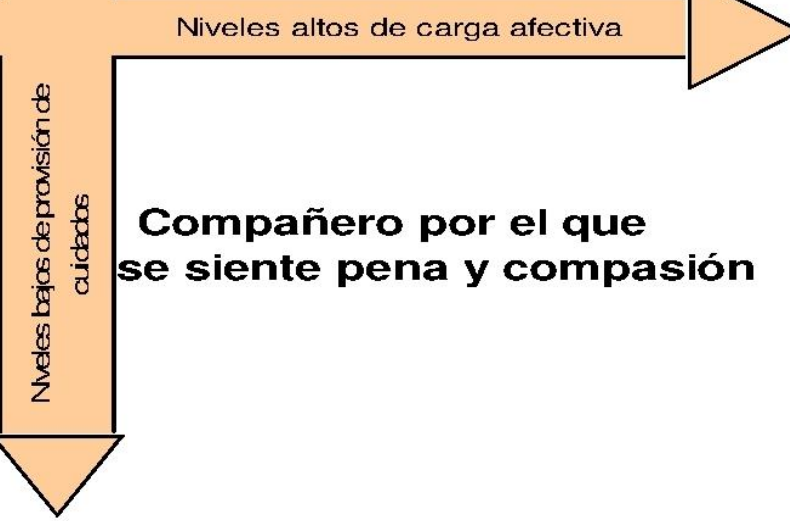

Niveles altos de carga afectiva-ejecución de cuidados entre los compañeros: de nuevo aparece el concepto de "hermano" y "hermana". Se trata de compañeros muy apegados emocionalmente, entre los que se establece una auténtica relación familiar (aunque no son familiares biológicamente) y de complicidad. La provisión de cuidados es mutua e incluye ayudarse en las actividades básicas de la vida diaria, alertar cuando el compañero está enfermo y compartir bienes materiales.

M7: "La compañera que tengo yo de cama, en el cuarto, ella entró tres días antes que yo y estamos como el primer día. Es una criatura también muy buena, muy buena, muy buena, como una hermana."

Niveles altos de carga afectiva-sin ejecución de cuidados entre compañeros: es un tipo de situación típica cuando conviven un residente válido con otro en situación de dependencia y/o demencia. Se trata de un compañero por el que se siente pena y 
compasión pero con el que no se llega a establecer una relación de cuidado, seguramente por que no puede ser establecida de forma recíproca.

Niveles bajos de carga afectiva-ejecución de cuidados entre compañeros: se trata de un compañero al que prácticamente se le considera como un objeto. La persona más válida ejerce cuidados de forma unilateral sobre él, pero en realidad es por ayudar y por complacencia con los cuidadores y con las cuidadoras.

Niveles bajos de carga afectiva-sin ejecución de cuidados entre compañeros: se trata de un compañero o compañera al que se denomina como "molesto" y con el que apenas hay interacción.

Los cuidadores y cuidadoras. Dimensionamos los conceptos de carga afectiva frente a la autoridad ejercida (Figura 3).

Figura 3. Los cuidadores o cuidadoras

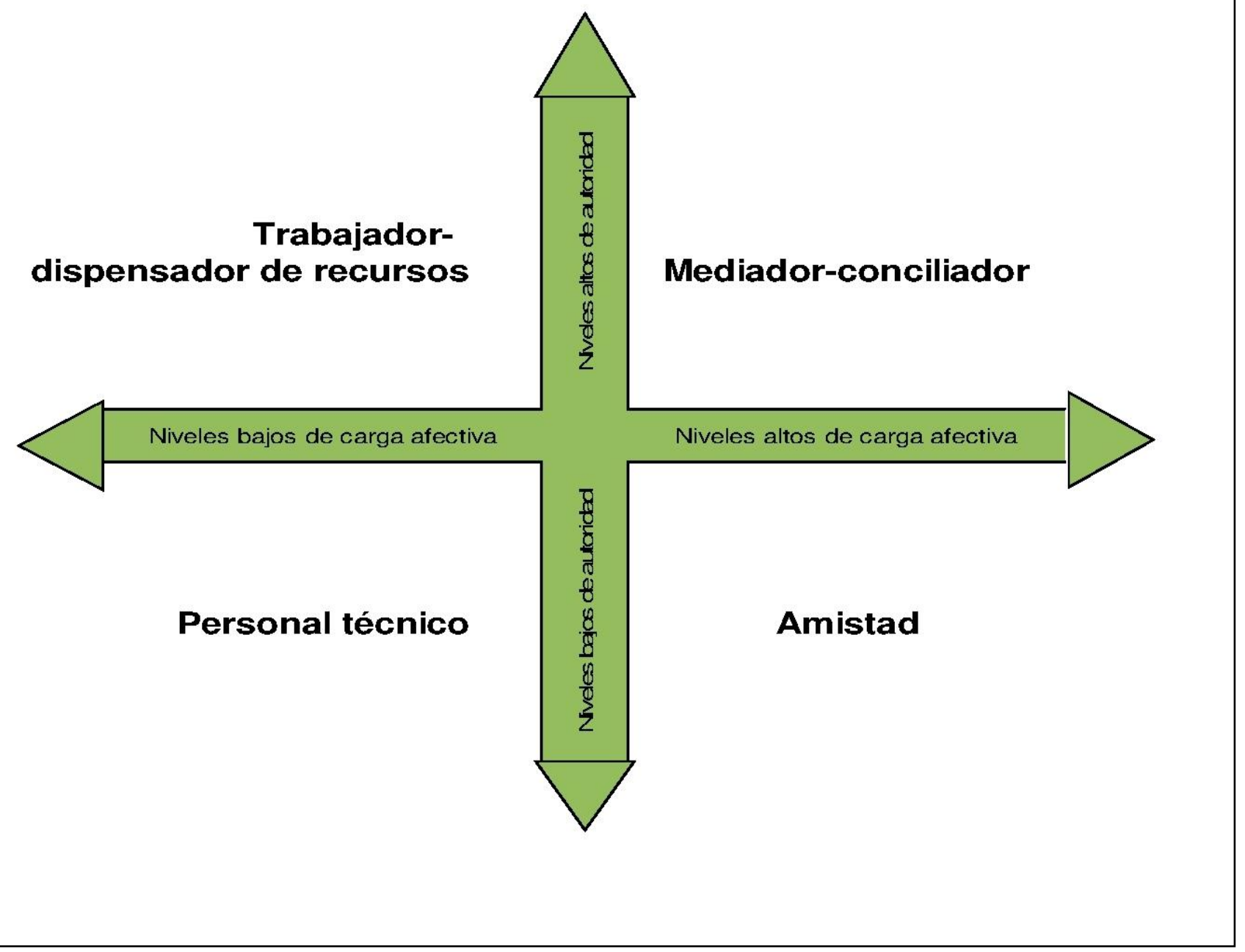

Niveles altos de carga afectiva-niveles altos de autoridad: los enfermeros y enfermeras y el resto de cuidadores ejerceríamos en este caso como mediadores y conciliadores, por ejemplo, para resolver conflictos entre los residentes, o para propiciar el establecimiento de relaciones sociales entre ellos. 
Niveles bajos de carga afectiva-niveles altos de autoridad: en este caso el cuidador actúa como un mero trabajador a las órdenes de la institución y como un dispensador y/o gestor de recursos para la persona mayor. Se trata de una relación típica que se establecería entre las personas mayores y el responsable de su centro (cuando no existe mucho contacto) o con el personal cuidador cuando se le solicita algún tipo de recurso material: por ejemplo, una silla nueva, o arreglar la TV que está estropeada.

M5: "Conchi es la que está de supervisora de aquí, que ésta anda mandándonos a todo el mundo que es lo que le gusta a ella y ella es la que manda cómo estamos, cómo no estamos y lo que nos hace falta y Amalia que es la directora y ya está."

Niveles altos de carga afectiva-niveles bajos de autoridad: se produce cuando el cuidador o la cuidadora son considerados prácticamente como un amigo o cuando los cuidados son ejercidos por ejemplo entre compañeros y compañeras de cuarto de forma recíproca.

Niveles bajos de carga afectiva-niveles bajos de autoridad: el cuidador en este caso es un mero técnico, que pone la medicación o hace la cura de una úlcera por presión, pero que prácticamente no interacciona con la persona mayor.

\section{DISCUSIÓN}

\section{Discusión de los resultados}

En el trabajo de Hauge y Heggen ${ }^{(3)}$, sobre el comportamiento de los residentes en los salones comunes, cuando se caracteriza físicamente a estas estancias se referencia la falta de objetos personales como fotografías, manteles bordados, cojines... Se indica que las relaciones sociales que se establecen entre las personas mayores en estas habitaciones son frágiles. En las residencias cordobesas también son escasas las charlas y las interacciones en este tipo de salas y sus moradores prefieren pasar el rato viendo la televisión. En ambos estudios, los residentes más independientes prefieren abandonar estas salas y moverse a lo largo de los corredores o acudir a sus cuartos propios.

Oh ${ }^{(4)}$, estudió el choque que supone la circunstancia de compartir habitación con un compañero o compañera afectados por demencia. Se trata ésta de una experiencia molesta intensa que afecta física y emocionalmente al conviviente lúcido y que supone también una disminución de sus interacciones sociales. En mi actual estudio también aparece reflejado este aspecto perturbador de la convivencia pero algunos y algunas residentes han dado la vuelta a esta situación y se han convertido en auténticos cuidadores de su compañero o compañera menos favorecido, lo que incluso ha contribuido a hacer mucho más significativa su vida diaria.

En el trabajo de Silva y cols. ${ }^{(2)}$, se evidencia cómo las relaciones de amistad establecidas en la residencia fueron más allá de las diferencias físicas, de edad o de cultura. Sin embargo, en nuestro centro de mayores, los residentes y las residentes tienden a mantener relaciones amistosas con aquellas personas pertenecientes a su mismo nivel social, en función de la educación o de las circunstancias económicas.

Algunos de los participantes y las participantes en el estudio de Bergland y Kirkevold ${ }^{(1)}$ y Nay ${ }^{(6)}$, expresan su deseo de establecer relaciones más profundas con los 
compañeros y compañeras o incluso relaciones de tipo sentimental, mientras que en nuestro trabajo los informantes y sobre todo las informantes sin pareja declaran abiertamente no buscar ni pretender establecer este tipo de interacciones. La formación de una pareja en una de las residencias donde se ha ejecutado este estudio, propició una serie de rumores y comentarios que originaron problemas entre diversos residentes, tal y como se alude en el estudio de Mendoza y cols. ${ }^{(5)}$, a que son los compañeros los que no respetan la propia intimidad y forma de vida. Para Bergland y Kirkevold ${ }^{(1)}$, el personal de enfermería tiene una labor facilitadora en el establecimiento de relaciones más intensas entre los compañeros o compañeras (sean de tipo amistoso o sentimental): en nuestro estudio se relata cómo las personas mayores acuden al personal en muchas ocasiones como confidentes en relación a estos temas.

Tanto en nuestro estudio como en el de Anderberg y Berglund ${ }^{(7)}$, se manifiesta por parte de los residentes la importancia de la comunicación y el trato cálido del personal cuidador. Los participantes suecos muestran más interés en los propios autocuidados que el que expresan mis entrevistados, los cuales mantienen un mayor nivel de dependencia emocional hacia los cuidados que les proporcionan otras personas, casi como una necesidad de llamar la atención y estar en continuo contacto con sus cuidadores.

En el estudio de Chang ${ }^{(8)}$, se expone como uno de los motivos por los que son importantes los autocuidados, es para evitar la necesidad de tener que estar demandando continuamente asistencia al personal. Como hemos visto también en nuestro trabajo y en otros, estos requerimientos de cuidados pueden producir conflictos y sentimientos negativos si no son atendidos todo lo rápido que las personas mayores pretenden o necesitan. El deseo de volver al hogar propio, así como la capacidad de proporcionar apoyo por parte de las personas cuidadoras y los familiares de las personas mayores, son algunos aspectos concurrentes en varios de estos estudios y que son identificados en el estudio de Chang como factores facilitadores de la realización de autocuidados.

Los informantes y las informantes del estudio de Liukkonen ${ }^{(9)}$ demandan que el personal dedique más tiempo a hablar con ellos: si bien los cuidadores y cuidadoras de nuestros centros investigados también están muy presionados por el tiempo, tienen perfectamente asumido entre sus roles el de proporcionar atención, charla y comprensión a sus pacientes.

\section{CONCLUSIÓNES}

Las personas que interaccionan en el centro desempeñan en relación al residente una serie de roles, los cuales podemos tratar de explicar dimensionando algunas de las cualidades más significativas de estas personas que se desenvuelven en la institución.

Las relaciones amistosas más intensas establecidas entre nuestros informantes, se consolidan entre personas que son percibidas como de un nivel similar dentro del estatus social, económico o educacional.

En numerosos casos en los que conviven en un mismo dormitorio una persona funcionalmente independiente con otra persona dependiente, la persona más válida 
asume espontáneamente (o por complacer a los cuidadores) el papel de cuidador informal de su compañero o compañera.

La relación con los cuidadores está determinada por una combinación entre la carga emocional que se establece entre los residentes con los profesionales y el nivel de autoridad que es percibido por los mayores en el citado personal cuidador.

\section{REFERENCIAS}

1. Bergland $A$, Kirkevold, $M$. The significance of peer relationships to thriving in nursing homes. Journal of Clinical Nursing 2008; 17 (10): 1295-302.

2. Silva CA, Menezes MR, Santos AC, Carvalho LS, Barreiros EX. Relacionamento de amizade na instituição asilar. Revista Gaúcha de Enfermagem 2006; 27 (2): 274-83.

3. Hauge S, Heggen K. The nursing home as a home: a field study of residents' daily life in the common living rooms. Journal of Clinical Nursing 2008; 17 (4): 460-7.

4. Oh J. Stroke patients' experiences of sharing rooms with dementia patients in a nursing home. International Journal of Nursing Studies 2006; 43 (7): 839-49.

5. Mendoza E, Martínez B, García M, Ullivarri N. Sexuality in institutionalized elderly people. Gerokomos 2005; 16 (2): 90-6.

6. Nay R. Sexuality and aged women in nursing homes. Geriatric Nursing 1992; 13 (6): 312-4.

7. Anderberg $\mathrm{P}$, Berglund AL. Elderly persons' experiences of striving to receive care on their own terms in nursing homes. International Journal of nursing practice 2010; $16(1): 64-8$.

8. Chang S. Beliefs about self-care among nursing home staff and residents in Taiwan. Geriatric Nursing 2009; 30 (2): 90-8.

9. Liukkonen A. Life in a nursing home for the frail elderly... including commentary by Anderson M.C. Clinical Nursing Research 1995; 4 (4): 358-72.

10. Strauss A, Corbin J. Bases de la Investigación Cualitativa. Técnicas y procedimientos para desarrollar la Teoría Fundamentada. Medellín: Universidad de Antioquia; 2002.

11. Taylor SJ, Bodgan R. Introducción a los métodos cualitativos de investigación. Barcelona: Paidós; 1982.

12. Amezcua M. El Trabajo de Campo Etnográfico en Salud. Una aproximación a la observación participante. Index de Enfermería (edición digital) 2000; 30. Disponible en: http://www.index-f.com/index-enfermeria/30revista/30 articulo 30-35.php

13. Carvalho Dantas C, Luzia Leite J, Soãres de Lima SB, Conceição Stipp MA. Teoría fundamentada en los datos-Aspectos conceptuales y operacionales: metodología posible de ser aplicada en la investigación en Enfermería. Revista latinoamericana Enfermagem 2009; 17 (4). Disponible en: http://www.scielo.br/scielo.php?script=sci arttext\&pid=S0104-

$11692009000400021 \& \operatorname{lng}=$ en\&nrm=iso\&tlng=es.

14. Soãres de Lima SB, Luzia Leite J, Erdmann AL, Prochnow AG, Conceição Stipp, MA, Lima García VR. La teoría fundamentada en datos: un camino a la investigación en Enfermería. Index de Enfermería (edición digital) 2010; 19 (1). Disponible en: http://www.index-f.com/index-enfermeria/v19n1/sumario.php

15. Emanuell E, Wendler D, Grady C. What makes clinical research ethical? JAMA 2000; 283, 2701-11.

16. Barrio Cantalejo IM, Simón Lorda P. Problemas éticos de la investigación cualitativa. Medicina Clínica 2006; 126 (11): 418-23. 
17. Morse JM, Barret M, Mayan M, Olson K, Spiers J. Verification strategies for establishing reliability and validity in qualitative research. International Journal of Qualitative Methods 2002; 2 (1): 13-22.

18. Castillo E, Vásquez ML. El rigor metodológico en la investigación cualitativa. Colombia Médica 2003; 34: 164-7.

19. De la Cuesta Benjumea C. La teoría fundamentada como herramienta de análisis. Cultura de los cuidados 2006; Año X (20): 136-40.

20. Glaser B. Theoretical Sensitivity: Advances in the methodology of Grounded Theory. CA Mill Valley: Sociology Press; 1978. 